\title{
Comment on "Nonmonotonic $d_{x^{2}-y^{2}}$ Superconducting Order Parameter in $\mathrm{Nd}_{2-x} \mathrm{Ce}_{x} \mathrm{CuO}_{4} "$
}

In a recent Letter 1] Blumberg et al. address the symmetry of the superconducting gap in cuprate superconductors. In particular in the electron-doped systems the issue is not yet settled, and not even the phase sensitive experiments [2] arrive at a consistent conclusion. Therefore more spectroscopic information is useful: in addition to the $\mathrm{B}_{1 g}$ and $\mathrm{B}_{2 g}$ Raman spectra [3] Blumberg and coauthors measured the $\mathrm{A}_{1 g}$ component by using an excitation energy of $1.9 \mathrm{eV}$. They obtain positions of the $\mathrm{A}_{1 g}, \mathrm{~B}_{1 g}$ and $\mathrm{B}_{2 g}$ pair breaking peaks at 45,50 and $67 \mathrm{~cm}^{-1}$, respectively, and conclude, from these positions alone, that the superconducting order parameter has $d_{x^{2}-y^{2}}$ symmetry with a non-monotonic dependence on the azimuthal angle $\phi$ (see Fig. 1b in Ref. [1]).

In this Comment we show that the basis for this conclusion is insufficient. This becomes already clear by just following the qualitative arguments of the authors: since the Raman scattering amplitudes $\gamma_{\mu}(\phi)$ of all symmetries $\mu$ are finite at the maximum $\Delta_{0}$ of the proposed gap function, the spectra in all symmetries will exhibit structures at the same energy $2 \Delta_{0}$ as opposed to what is observed 1, 3]. In addition, if $\Delta(\phi)$ has components up to $\sin (10 \phi)$ (see caption of Fig. 1) as proposed in Ref. 1] it is hard to probe them with $\gamma_{\mu} \propto \sin (2 \phi)$ without applying a model. Therefore, we calculated the Raman response explicitly. [4] For a small phenomenological damping $\Gamma / \Delta_{0}=0.04$ (Fig. 1 a) both the $B_{1 g}$ and the $B_{2 g}$ spectra exhibit two structures at around 50 and $67 \mathrm{~cm}^{-1}$, and the $A_{1 g}$ peak is at approximately 60 and not at $45 \mathrm{~cm}^{-1}$ as in the experiment. Even for this small damping all spectra are linear (see inset of Fig. 10 a) up to approximately $20 \mathrm{~cm}^{-1}$ [4]. A larger damping $\Gamma / \Delta_{0}=0.30$ (Fig. 11 b) not only smears out the double peak structure but also completely kills the power laws characteristic for $d$-wave pairing for this type of gap. In general, neither the $\mathbf{k}$ dependence nor the magnitude of the gap can be derived from the peak positions alone. Rather an appropriate model and the complete study of the low-temperature and low-frequency power laws are required. Then, constraints for the gap similar to those from the specific heat or the magnetic penetration depth could be obtained.

Here, it is indeed the magnetic penetration depth 5 $\lambda(T)$ which is in direct conflict with the proposed form of the gap. The temperature dependence of $\lambda(T)$ can be readily calculated from the functional dependence $\Delta(\phi)$. No agreement with the data [5] at any doping can be achieved. Supposed the nonmonotonic gap would be realized an analysis with a monotonic one [5] would lead to $\Delta_{0} \simeq 9 k_{B} T_{c}$ in spite of the restricted phase space around the node.

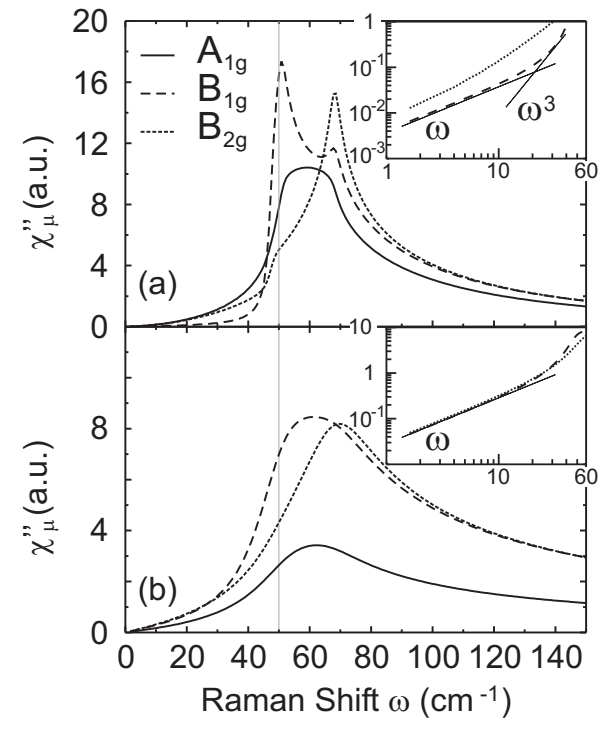

FIG. 1: Raman response calculated with the gap function proposed in Ref. [1]. The best analytical approximation is given by $\Delta(\phi)=\Delta_{0}\left[\sin (2 \phi)+a_{1} \sin (6 \phi)+a_{2} \sin (10 \phi)\right]\left(2 \Delta_{0}=\right.$ $\left.67 \mathrm{~cm}^{-1}, a_{1}=0.42, a_{2}=0.17\right)$ using the same definition of $\phi$ as in Ref. [1]. In this nomenclature the scattering amplitudes read: $\gamma_{B_{1 g}} \propto \sin (2 \phi), \gamma_{B_{2 g}} \propto \cos (2 \phi)$, and $\gamma_{A_{1 g}} \propto-\cos (4 \phi)$. Neither for small (a) nor for large (b) damping the power laws, in particular the $\omega^{3}$ dependence in $B_{1 g}$ symmetry can be observed any more for $\omega<20 \mathrm{~cm}^{-1}$ (insets).

In conclusion, the functional dependence of the gap proposed in Ref. [1] is neither sufficiently supported by the Raman results nor compatible with the magnetic pentration depth. In spite of that, an anisotropic $s$-wave as suggested earlier [3] is probably not the full story either at least not in the entire doping range. Therefore the issue of the superconducting gap in the electrondoped systems cannot at all be considered solved by now.

F. Venturini and R. Hackl, Walther Meissner Institute, Bavarian Academy of Sciences, 85748 Garching, Germany

U. Michelucci, EKM, Universität Augsburg, 86135 Augsburg, Germany

[1] G. Blumberg et al., Phys. Rev. Lett. 88, 107002 (2002).

[2] L. Alff et al., Phys. Rev. Lett. 83, 2644 (1999); C.C. Tsuei, and J.R. Kirtley, Phys. Rev. Lett. 85, 182 (2000).

[3] B. Stadlober et al., Phys. Rev. Lett. 74, 4911 (1995).

[4] T.P. Devereaux and D. Einzel, Phys. Rev. B, 51, 16336 (1995); T.P. Devereaux, Phys. Rev. Lett. 74, 4313 (1995); 
F. Venturini et al., Phys. Rev. B, 62, 15204 (2000).

207005 (2002).

[5] J.D. Kokales et al., Phys. Rev. Lett. 85, 3696 (2000); J.A. Skinta et al., Phys. Rev. Lett. 88, 207003 (2002) and ibid, 